Development of a Patient and Institutional-Based Model for Estimation of Operative Times for Robot-Assisted Radical Cystectomy: Results from the International Robotic

\title{
Cystectomy Consortium
}

Ahmed A. Hussein ${ }^{1,2}$, Paul R. May ${ }^{1}$, Youssef E. Ahmed ${ }^{1}$, Matthias Saar ${ }^{3}$, Carl J Wijburg ${ }^{4}$, Lee Richstone $^{5}$, Andrew Wagner ${ }^{6}$, Timothy Wilson ${ }^{7}$, Bertram Yuh $^{7}$, Joan Palou Redorta ${ }^{8}$, Prokar Dasgupta $^{9}$, Omar Kawa ${ }^{9}$, Mohammad Shamim Khan ${ }^{9}$, Mani Menon ${ }^{10}$, James O. Peabody ${ }^{10}$, Abolfazl Hosseini ${ }^{11}$, Franco Gaboardi ${ }^{12}$, Giovannalberto Pini ${ }^{12}$, Francis Schanne ${ }^{13}$, Alexandre Mottrie $^{14}$, Koon-ho Rha ${ }^{15}$, Ashok Hemal ${ }^{16}$, Michael Stockle ${ }^{3}$, John Kelly ${ }^{17}$, Wei Shen Tan ${ }^{17}$, Thomas J. Maatman ${ }^{18}$, Vassilis Poulakis ${ }^{19}$, Jihad Kaouk ${ }^{20}$, Abdullah Erdem Canda ${ }^{21}$, Mevlana Derya Balbay $^{21}$, Peter Wiklund ${ }^{11}$, Khurshid A. Guru ${ }^{1}$

${ }^{1}$ Roswell Park Cancer Institute, Buffalo, NY, ${ }^{2}$ Cairo University, Egypt, ${ }^{3}$ University of the Saarland, Homburg Saar, Germany, ${ }^{4}$ Rijnstate Hospital, Arnhem, the Netherlands, ${ }^{5}$ The Arthur Smith Institute for Urology, New York, NY, ${ }^{6}$ Beth Israel Deaconess Medical Center, Boston, MA, ${ }^{7}$ City of Hope and Beckman Research Institute, Duarte, CA, ${ }^{8}$ Fundacio Puigvert, Barcelona, Spain, ${ }^{9}$ Guy's Hospital and King's College London School of Medicine, London, UK,

${ }^{10}$ Henry Ford Health System, Detroit, MI, ${ }^{11}$ Karolinska University Hospital, Stockholm, Sweden, ${ }^{12}$ San Raffaele Turro Hospital, Milan, Italy, ${ }^{13}$ Urological Surgical Associates of Delaware, DE., ${ }^{14}$ Onze-Lieve-Vrouw Ziekenhuis, Aalast, Belgium, , ${ }^{15}$ Yonsei University Health System Severance Hospital, Seoul, Korea, ${ }^{16}$ Wake Forest University Baptist Medical Center, Winston-Salem, NC, ${ }^{17}$ Division of Surgery and Interventional Science, University College London, Department of Urology, ${ }^{18}$ Michigan Urological Clinic, Grand Rapids, MI., ${ }^{19}$ Doctor's Hospital of Athens, Athens, Greece ${ }^{20}$ Glickman Urological and Kidney Institute, Cleveland Clinic, $\mathrm{OH},{ }^{21}$ Yildirim Beyazit University, School of Medicine, Ankara Ataturk Training and Research Hospital, Ankara, Turkey, 


\section{Corresponding Author:}

Khurshid A. Guru, MD

Elm \& Carlton St Buffalo, NY 14263

Tel: 716-845-4155;

Fax: $716-845-3300$

Khurshid.guru@ roswellpark.org

Professor of Oncology

Department of Urology, Roswell Park Cancer Institute

A.T.L.A.S (Applied Technology Laboratory for Advanced Surgery) Program

Keywords: Robot-assisted, cystectomy, operative time, quality control, scheduling, 


\begin{abstract}
Introduction: Operative times can be accurately predicted with feedback and knowledge of the key variables. Our study aimed to utilize the International Robotic Cystectomy Consortium (IRCC) database of robot-assisted radical cystectomy (RARC) to determine patient and institutional variables of importance in predicting operative times.
\end{abstract}

Methods: IRCC database includes 2686 RARCs performed at 23 institutions from 12 countries. The model included variables that are available in the preoperative period used and therefore can be used for prediction of surgical times: institutional RARC volume, age at RARC, gender, BMI, ASA Score, history of prior abdominal surgery and radiation, clinical stage of disease, administration of neoadjuvant chemotherapy, type and technique of diversion and the extent of pelvic lymph node dissection. A conditional inference tree method was used to fit a binary decision tree predicting operative time. Permutation tests were performed to determine the variables having the strongest association with RARC surgical time. The data was split at the value of this variable resulting in the largest difference in means for the surgical time across the split. This process was repeated recursively/iteratively on the resultant data sets until the permutation tests showed no significant association between any of the explanatory variables and operative time.

Results: 2135 procedures were included in the analysis. The variable most strongly associated with surgical time was the type of diversion (ileal conduits - 69 minutes shorter than neobladders, $\mathrm{p}<0.001)$. Among patients who received neobladders, type of lymph node dissection (LND) was also strongly associated with surgical time. Among the Ileal conduit patients, institutional surgeon volume (>66 RARCs) was an important factor (higher volume54 minutes shorter, $\mathrm{p}<0.001)$. The regression tree output was in the form of box plots that show 
the median, interquartile deviation, and ranges of surgical times according to the patient, disease and institutional characteristics.

Conclusion: We developed a methodology utilizing a large database to estimate operative times for RARC based on patient, disease and institutional metrics. 


\section{Introduction}

An operating room (OR) is considered to be one of the most costly functional areas within hospitals as well as its major profit center. It is known that managing an OR department is a challenging task, which requires the integration of many actors (e.g., patients, surgeons, nurses, technicians) who may have conflicting interests and priorities. Considering these aspects, this paper focuses on developing a methodology for scheduling operating rooms that reflects the complexity, and variability associated with surgery.

Radical cystectomy (RC) with urinary diversion is a complex surgery associated with significant morbidity and cost ${ }^{1}$. RCs performed with robot-assistance has grown dramatically (<1\% to $13 \%$ ) between 2004 and $2010^{2}$. Despite the benefits of robot-assisted radical cystectomy (RARC) in terms of perioperative outcomes such as blood loss, hospital stay and recovery, it has been criticized for long operative times and the associated cost. Although RARC was associated with shorter hospital stay ( $\$ 658$ a day) when compared to open RC, it was also associated with significantly longer operative times (\$ 1902 an hour). Continuous refinement of the technique and expertise may cause additional cutting down of operative times and costs ${ }^{3}$.

For RC, prolonged operative times have been associated with higher incidence of complications and perioperative mortality independent of the disease stage or associated comorbidities ${ }^{4}$. Additionally, longer operative times have been directly associated with increased healthcare costs, where each operating room (OR) minute was found to add $\$ 15$ to the

overall hospital charges ${ }^{5}$. Not surprisingly, operative time has been identified as a quality measure for surgical performance for RARC ${ }^{6,7}$. 
Scheduling OR time for RARC is a challenging task owing to the complexity and reconstructive nature of the procedure. Patients have multiple confounding factors that contribute to variation in operative time for RARC, including patient demographics and comorbidities, disease stage, procedural complexity, technical modifications, surgeon experience and hospital volume $^{8}$. In this context, we sought to develop a statistical model that incorporates different preoperative data, including patient, disease, surgical and institutional variables, to estimate operative times for RARC at the individual patient level.

\section{Methods:}

A retrospective review of 2686 RARCs performed at 23 institutions from 12 countries included in the International Robotic Cystectomy Consortium (IRCC) database (I-97906) was performed. For prediction of operative time (from incision to wound closure), we included all the relevant patient, disease, technical and institutional variables that can be assessed preoperatively and therefore can be included in a predictive model. Patient factors included: age, gender, body mass index (BMI), the American Society of Anesthesiologists (ASA) score, prior history of abdominal surgery or irradiation. Disease factors included preoperative clinical staging. Technical factors included the receipt of neoadjuvant chemotherapy, planned type and technique of diversion, and extent of pelvic lymph node dissection (pLND). The overall RARC institutional volume was also included in the model.

Descriptive statistics were used to summarize the data. A conditional-inference tree method was used to fit a binary decision tree predicting the distribution of operative times. Permutation tests were performed to determine the variable having the strongest association with RARC surgical time. The data was split at the value of this variable resulting in the largest 
difference in means for the surgical time across the split This process was repeated recursively on the resultant data sets until the permutation tests showed no significant association between any of the explanatory variables and operative time. The resulting data sets are known as terminal nodes or leaves.

The output of the software package was in the form of box plots depicting the median, interquartile ranges, the minimum and the maximum duration of operative times within each terminal node. Operative times are generally known to be lognormally distributed ${ }^{9}$. Within each terminal node a lognormal model was also fit to the operative times of patients included in the node. This lognormal model fit allows any quantity associated with the distribution of operative times to be estimated.

All tests were two-sided, with statistical significance defined as $p<0.05$. All statistical

analyses were performed using R software (version 3.2, R Core Team (2016). R: A language and environment for statistical computing (R Foundation for Statistical Computing, Vienna, Austria. URL https://www.R-project.org/).

\section{Results}

The final analysis comprised 2135 RARCs (Table 1). Mean age was 67 years (standard deviation [SD] 10), 74\% were males. Sixteen percent had clinical extravesical disease and 20\% received neoadjuvant chemotherapy. Seventy eight percent received ileal conduits and $69 \%$ had an intracorporeal diversion. Median operative time was 364 minutes (Interquartile range [IQR] 300-447). Fifty seven percent had extended, 35\% had standard LND and 39\% of patients had $\geq 20$ lymph nodes on the final pathology (Table 1). There was a trend towards shorter operative 
times for RARC, decreasing from a mean of 373 minutes in 2006 to 323 minutes in 2015 $(\mathrm{p}=0.052)($ Figure 1$)$.

The variable most strongly associated with surgical time was the type of diversion and it resulted in the largest mean difference (ileal conduit - 69 minutes shorter than neobladders, $\mathrm{p}<0.001)$. Among patients who received neobladders, the extent of LND was the most strongly associated with RARC time. Extended or standard LNDs were 26 minutes on average longer than limited or no LND dissection $(\mathrm{p}<0.001)$. Whatever the extent of LND, having a lower BMI was significantly associated with at least 33 minutes shorter operative time (Figure 2).

Among patients who received ileal conduits, surgeon volume $>66$ procedures was significantly associated with shorter operative times (56 minutes, $\mathrm{p}<0.001)$. Again, whatever the surgeon volume was, lower BMI was significantly associated with shorter operative times $(\mathrm{p}<0.001)$. For lower volume surgeons (<66 RARCs) and BMI $\leq 30 \mathrm{~kg} / \mathrm{m}^{2}$, the extent of LND further affected operative time (20 minutes longer in extended or standard LND, p<0.001). For patients who underwent standard or extended pLND, prior abdominal surgery and surgeon volume (41 RARCs) were also significantly associated with shorter operative times $(\mathrm{p}<0.001)$ (Figure 2).

The longest estimated operative times were observed in patients who received neobladders and underwent limited or no LND and with BMI > $41 \mathrm{~kg} / \mathrm{m}^{2}$ (Node 5; median 461 minutes, IQR 390-571 minutes). On the other hand, the shortest estimated operative times were patients who received ileal conduits, had BMI $\leq 30 \mathrm{~kg} / \mathrm{m}^{2}$, underwent standard or extended LND, did not have any prior surgery and RARC performed surgeons with volume 42-66 RARCs (Node 16: median 284, IQR 264-340 minutes) (Figure 2) (Table 2). 
The use of the binary decision tree is best illustrated with an example. Assume a urologist who has performed 50 RARCs had a patient whose BMI is $26 \mathrm{Kg} / \mathrm{m}^{2}$ and no prior abdominal surgery. The patient is to be scheduled for a RARC with an ileal conduit and extended LND. Starting at the top of the tree(Figure 2), we proceed to the right due to the scheduled ileal conduit. Then, at node 9 we proceed left due to the surgeon's volume of 25 RARCs. At node 10 we proceed left due to the patient's BMI. At node 11 we again proceed left due to the planned extended LND. At node 12 we proceed to the right because the patient has no history of abdominal surgery. Finally we proceed right, again due to the surgeon experience, ending in node 16. From Table 2 we can now see that similar surgeries had a mean operative time of 307 minutes (SD 60 minutes). Similarly the minimum, maximum, median and IQR for similar surgeries is readily available in Table 2 .

\section{Discussion}

Bladder cancer is one of the most expensive cancers to manage ${ }^{1,10}$. Bearing this in mind, it is vital to explore the association between patient, disease, surgeon, and institutional factors with RC operative times. Within hospitals, ORs have been identified as the key financial component, where they contribute to more than $40 \%$ of hospitals' revenue ${ }^{11}$. On the other hand, costs associated with staffing and equipment rendered OR utilization very expensive, accounting

for approximately $30 \%$ of the total hospital expenditure ${ }^{12}$. Late starting or finishing times and large time gaps between surgeries can lead to suboptimal OR utilization. Consequently, attempts are made to optimize the OR availability to maximize profitability, minimize expenditure (costs associated with staffing, especially the overtime cost), and limit under-utilization. 
Scheduling operative times can be done via various methods. Common strategies include: open (assigning an OR at the convenience of surgeons), block scheduling (surgeons are assigned time blocks into which they arrange their procedures) and modified block scheduling (some time is blocked and some is left open and any unused time can be released) ${ }^{13}$. The key to maximizing OR utilization is to determine the appropriate block time for each kind of surgery, considering the different variables that may affect it. Accurate estimates of operative times would facilitate scheduling, service planning and maximize the utility of the OR. Historical data, such as the average time for the last ten cases, average surgeon's list or surgeon's estimate have been proposed as means for estimating scheduled operative times ${ }^{14}$. However, none of these methods have shown a reliable predictive validity ${ }^{14}$. As a result, OR utilization can be as low as $80 \%$ of the target, which has substantial financial implications ${ }^{15}$.

Surgery planning and scheduling offers unique challenges owing to the amount of associated uncertainty. It requires integration of multiple and variable factors, including patient, surgical team, disease, technical, surgeon and institutional factors. Not only that, each patient is unique and therefore, even for the same procedure, the scheduled time for one patient may not be appropriate for another. Different statistical methods have been proposed, including linear regression, generalized linear, and intelligent-based models ${ }^{9}$. Selection of a model should be based on examination of the data distribution, where linear regression can be used in cases of normal distribution. Recently, there has been a trend towards incorporating intelligent-based models and data mining techniques such as rough sets, artificial neural networks and fuzzy inference systems to predict procedure times, despite initial unsatisfactory results ${ }^{16}$. We utilized a multi-level conditional-inference tree model that can handle complex interactions between variables, and determine the contribution of each variable at each level. Tree-based models have 
several advantageous features including scalability to large numbers of explanatory variables and subjects, simplicity of model interpretation and ease of use by the non-statistician. These models are also adept at fitting data that is far from normally distributed. Utilizing this model we were able to estimate operative times at the individual patient level.

Filson et al examined the different factors that may contribute to operative times. They divided them into potentially modifiable (such as perioperative procedures, LND, and diversion type and technique), non-modifiable patient factors (such as age and sex) and institutional and surgeon factors ${ }^{8}$. Similar to our study, they observed longer operative times with neobladders and with more extensive LNDs. Older age and the number of comorbidities were significantly associated with shorter operative times ${ }^{17}$. Surgeons are usually concerned of the potential higher anesthetic complications in older and sicker patients. Female patients were also found to have longer operative times (possibly because of performance of hysterectomy and vaginal reconstruction) ${ }^{8}$. This is in contrast to the current and prior studies ${ }^{18}$. BMI and prior abdominal surgery were significantly associated in our study with operative times. Higher BMI and prior abdominal surgery add to the complexity of RARC, with more time spent in port placement, careful dissection as well as LND ${ }^{17,19}$.

High-volume institutions had shorter operative times for RC. This may be attributed to the experience of the surgeon and the team at those institutions ${ }^{8}$. Institutional volume, however, was not significantly associated with operative times in our study. This may be explained by the fact that the IRCC includes mainly high volume institutions, which limits any conclusions drawn about the institutional volume. Other studies have shown clear association with hospital type, where academic centers had longer operative times (approximately 40 minutes longer). Academic centers involve postgraduate trainees (residents and fellows in anesthesia and 
urology), and they are also more likely to perform extended LNDs, intracorporeal diversion and neobladders ${ }^{20}$. In agreement with our findings, a significant decrease in operative time was associated with higher surgeon volume ${ }^{21,22}$. The cut off for surgical proficiency for RARC is higher in our study than previously reported (22 RARCs) ${ }^{23}$. In our study, surgeons who had performed at least 66 RARCs saved an average of 54 minutes of operative time among patients who underwent ileal conduits (Nodes 10 (mean 396 minutes) vs 19 (mean 340 minutes), $\mathrm{p}<0.001)$. A surgeon volume of 44 RARCs resulted in a savings of 77 minutes on average for patients who underwent extended LND (Nodes 15 (384 minutes) vs 16 (307 minutes), p<0.001). This highlights the importance of fellowship training and dedicated cystectomy programs where surgeons can increase their RARC volume ${ }^{24,25}$.

Despite the uniqueness of this study, several limitations exist. The retrospective study design has its recognized limitations. Any surgical procedure typically involves three stages: presurgery, surgery and post-surgery. The actual procedural duration (time elapsed from incision to wound closure) is the amount of time during which surgery occurs and corresponds to the defined Current Procedure Terminology (CPT) codes. Most databases do not account for nonoperative times that include delays in patient arrival, times related to anesthesia induction, patient discharge and turnover times (cleaning and preparing the OR for the next patient). Although the actual operative time would likely be shorter than the overall OR time, a reliable OR schedule can only be achieved when accurate estimates about the time needed to perform the surgery is available ${ }^{26}$. Otherwise, operations that take significantly longer or shorter than predicted will increase the chance of OR underutilization. We believe that some variability between scheduled and actual procedures cannot be avoided, especially those arising due to unexpected intraoperative findings. Another limitation of our study was the inability to account 
for the heterogeneity of teams and ORs due to the multi-institutional nature of the IRCC database.

\section{Conclusion}

We developed a methodology utilizing a large database to estimate operative times for RARC based on patient, disease and institutional metrics. 
Table 1. Perioperative outcomes of 2135 patients who received RARC

\begin{tabular}{|c|c|}
\hline Variable & Value \\
\hline Number of patients, $n$ & 2135 \\
\hline \multicolumn{2}{|c|}{ Preoperative characteristics } \\
\hline Age at cystectomy, mean (SD) (years) & $67(10)$ \\
\hline Sex, Males, n (\%) & $1578(74)$ \\
\hline BMI, mean (SD) $\left(\mathrm{kg} / \mathrm{m}^{2}\right)$ & $28(5)$ \\
\hline ASA score, mean (SD) & $2.4(0.7)$ \\
\hline Prior abdominal/pelvic surgery, $\mathrm{n}(\%)$ & $580(46)$ \\
\hline $\mathrm{NAC}, \mathrm{n}(\%)$ & $400(20)$ \\
\hline Clinical T stage $\geq 3, \mathrm{n}(\%)$ & $302(16)$ \\
\hline \multicolumn{2}{|l|}{ Operative outcomes } \\
\hline Type of diversion, Ileal conduit, $\mathrm{n}(\%)$ & $1553(78)$ \\
\hline Location of diversion, Intracorporeal, $\mathrm{n}(\%)$ & $1006(69)$ \\
\hline Operative time, median (IQR) (minutes) & $364(300-447)$ \\
\hline EBL, median (IQR) (ml) & $300(200-500)$ \\
\hline No LND, n (\%) & $103(9)$ \\
\hline Limited LND, n (\%) & $9(1)$ \\
\hline Standard LND & $412(35)$ \\
\hline Extended LND, n (\%) & $666(57)$ \\
\hline \multicolumn{2}{|l|}{ Pathologic outcomes } \\
\hline pT3/T4, n (\%) & $776(39)$ \\
\hline LNY, mean (SD) & $18.4(11)$ \\
\hline LNY $\geq 20, \mathrm{n}(\%)$ & $726(39)$ \\
\hline $\mathrm{N} 1, \mathrm{n}(\%)$ & $499(23)$ \\
\hline Positive soft tissue surgical margins, n (\%) & $144(7)$ \\
\hline \multicolumn{2}{|l|}{ Postoperative outcomes } \\
\hline Adjuvant chemotherapy, n (\%) & $262(16)$ \\
\hline Hospital stay, median (IQR) (months) & $9(6-13)$ \\
\hline ICU stay, median (IQR) & $1(0-1)$ \\
\hline Complications within 30 days, $\mathrm{n}(\%)$ & $559(26)$ \\
\hline Complications $\geq$ Clavien 3 within 30 days, $\mathrm{n}(\%)$ & $164(8)$ \\
\hline Complications within 90 days, $\mathrm{n}(\%)$ & $653(31)$ \\
\hline Complications $\geq$ Clavien 3 within 90 days, $\mathrm{n}(\%)$ & $202(10)$ \\
\hline Readmissions within 30 days, $\mathrm{n}(\%)$ & $115(5)$ \\
\hline Readmissions within 90 days, $\mathrm{n}(\%)$ & $197(9)$ \\
\hline Mortality within 30 days, $\mathrm{n}(\%)$ & $14(1)$ \\
\hline Mortality within 90 days, $\mathrm{n}(\%)$ & $51(3)$ \\
\hline Follow up, median (IQR) (months) & $12.4(5-27)$ \\
\hline \multicolumn{2}{|c|}{$\begin{array}{l}\text { RARC, robotic-assisted radical cystectomy; n, number; SD, standard } \\
\text { deviation; BMI, body mass index; } \mathrm{Kg} / \mathrm{m}^{2} \text {, kilogram per meter squared; ASA } \\
\text { score, American Society of Anesthesiologists; LN, lymph node; NAC, } \\
\text { neoadjuvant chemotherapy; LND; lymph node dissection; EBL, estimated } \\
\text { blood loss; LNY, lymph node yield }\end{array}$} \\
\hline
\end{tabular}


Table 2. Median, IQR, mean, SD and ranges for operative times for each Node.

\begin{tabular}{|c|c|c|c|c|c|c|c|c|}
\hline Node & $\begin{array}{c}\text { Patients } \\
\text { at Node } \\
\text { (n) }\end{array}$ & $\begin{array}{c}\text { Mean } \\
\text { operative } \\
\text { time } \\
\text { (minutes) }\end{array}$ & $\begin{array}{c}\text { SD } \\
\text { (minutes) }\end{array}$ & $\begin{array}{c}\text { Minimum } \\
\text { operative } \\
\text { time } \\
\text { (minutes) }\end{array}$ & $\begin{array}{c}\mathbf{2 5}^{\text {th }} \\
\text { percentile } \\
\text { (minutes) }\end{array}$ & $\begin{array}{c}\text { Median } \\
\text { operative } \\
\text { time } \\
\text { (minutes) }\end{array}$ & $\begin{array}{c}\mathbf{7 5}^{\text {th }} \\
\text { percentile } \\
\text { (minutes) }\end{array}$ & $\begin{array}{c}\text { Maximum } \\
\text { operative } \\
\text { time } \\
\text { (minutes) }\end{array}$ \\
\hline $\mathbf{4}$ & 326 & 438 & 114 & 157 & 375 & 434 & 501 & 760 \\
\hline $\mathbf{5}$ & 40 & 471 & 114 & 260 & 390 & 461 & 571 & 680 \\
\hline $\mathbf{7}$ & 74 & 390 & 93 & 159 & 345 & 375 & 448 & 600 \\
\hline $\mathbf{8}$ & 62 & 446 & 100 & 240 & 375 & 444 & 495 & 720 \\
\hline $\mathbf{1 3}$ & 59 & 379 & 99 & 210 & 308 & 350 & 435 & 618 \\
\hline $\mathbf{1 5}$ & 51 & 384 & 63 & 240 & 342 & 389 & 417 & 600 \\
\hline $\mathbf{1 6}$ & 21 & 307 & 60 & 239 & 264 & 284 & 340 & 440 \\
\hline $\mathbf{1 7}$ & 415 & 390 & 108 & 200 & 313 & 370 & 457 & 862 \\
\hline $\mathbf{1 8}$ & 166 & 430 & 108 & 200 & 360 & 420 & 490 & 780 \\
\hline $\mathbf{2 0}$ & 715 & 332 & 89 & 159 & 270 & 318 & 378 & 827 \\
\hline $\mathbf{2 1}$ & 205 & 371 & 100 & 172 & 300 & 360 & 420 & 830 \\
\hline
\end{tabular}

Figure 1. Mean operative times for RARC between 2005 and 2015 ( $\mathrm{p}=0.052)$

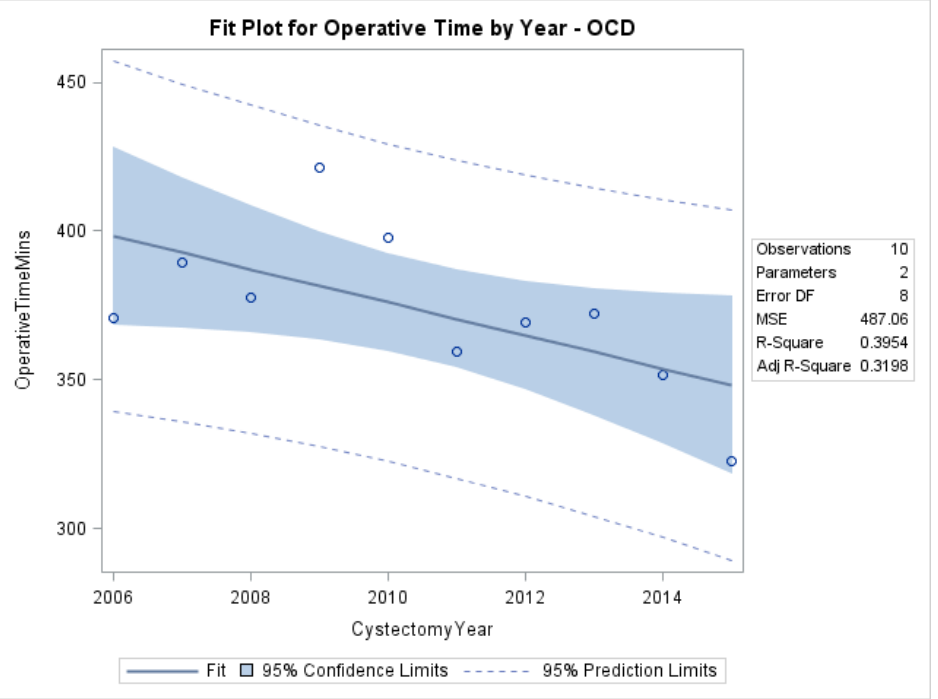


Figure 2. Regression tree showing the outcome as Box plots for each Node.

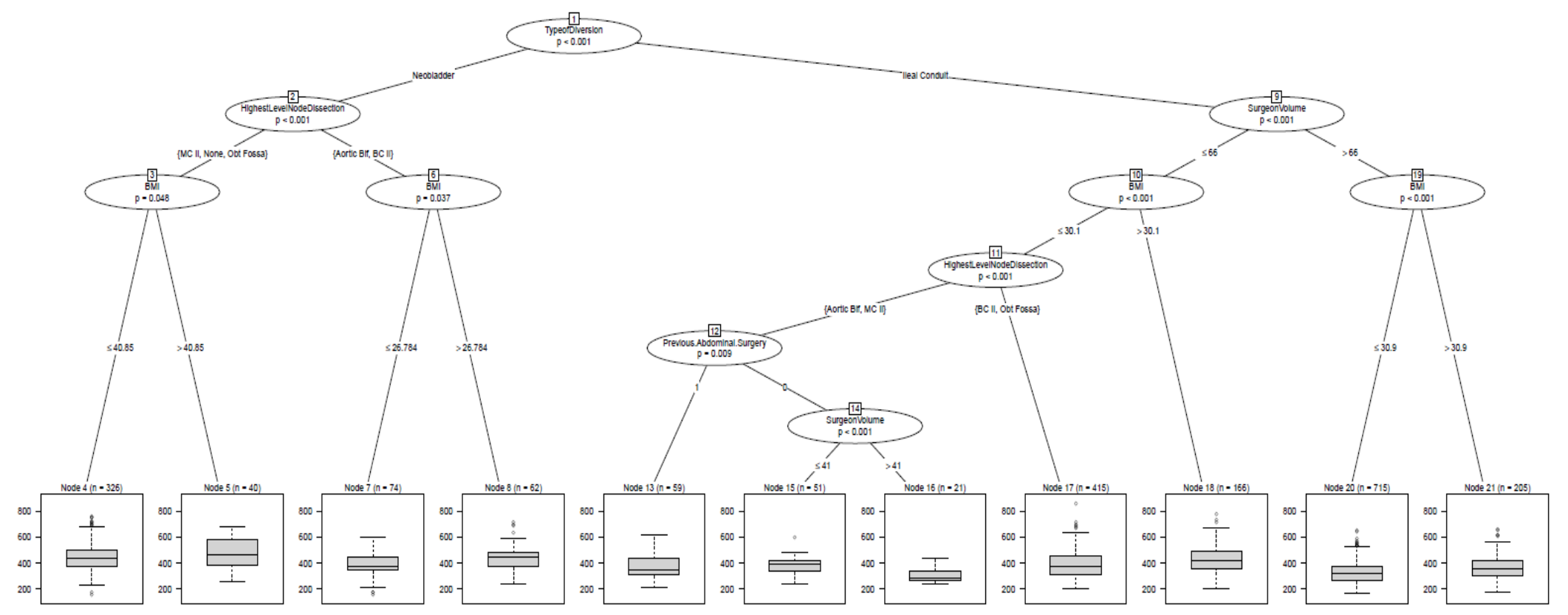

\section{References}

1. Svatek RS, Hollenbeck BK, Holmang $S$, et al. The economics of bladder cancer: costs and considerations of caring for this disease. Eur Urol. 2014;66(2):253-262.

2. Leow JJ, Reese SW, Jiang W, et al. Propensity-matched comparison of morbidity and costs of open and robot-assisted radical cystectomies: a contemporary population-based analysis in the United States. Eur Urol. 2014;66(3):569-576.

3. Smith A, Kurpad R, Lal A, Nielsen M, Wallen EM, Pruthi RS. Cost analysis of robotic versus open radical cystectomy for bladder cancer. J Urol. 2010;183(2):505-509.

4. Lavallee LT, Schramm D, Witiuk K, et al. Peri-operative morbidity associated with radical cystectomy in a multicenter database of community and academic hospitals. PLoS One. 2014;9(10):e111281.

5. Macario A. What does one minute of operating room time cost? J Clin Anesth. 2010;22(4):233236.

6. Hussein AA, Dibaj S, Hinata N, et al. Development and Validation of a Quality Assurance Score for Robot-assisted Radical Cystectomy: A 10-year Analysis. Urology. 2016. 
7. Wilson TG, Guru K, Rosen RC, et al. Best practices in robot-assisted radical cystectomy and urinary reconstruction: recommendations of the Pasadena Consensus Panel. Eur Urol. 2015;67(3):363-375.

8. Filson CP, Tan HJ, Chamie K, Laviana AA, Hu JC. Determinants of radical cystectomy operative time. Urol Oncol. 2016;34(10):431 e417-424.

9. Paola DM. A Methodology for Scheduling Operating Rooms Under Uncertainty: Industrial and Management Systems Engineering, University of South Florida; 2013.

10. Yeung $\mathrm{C}$, Dinh T, Lee J. The health economics of bladder cancer: an updated review of the published literature. Pharmacoeconomics. 2014;32(11):1093-1104.

11. Guerriero F, Guido R. Operational research in the management of the operating theatre: a survey. Health Care Manag Sci. 2011;14(1):89-114.

12. Vargas LG, May JH, Spangler W, Stanciu A, Strum DP. Operating Room Scheduling and Capacity Planning. London: Springer; 2008.

13. Fei H, Meskens $\mathrm{N}$, Chu C. A planning and scheduling problem for an operating theatre using an open scheduling strategy. Comput. Ind. Eng. 2010;58(2):221-230.

14. Strum DP, Sampson AR, May JH, Vargas LG. Surgeon and type of anesthesia predict variability in surgical procedure times. Anesthesiology. 2000;92(5):1454-1466.

15. Achieving operating room efficiency through process integration. Healthcare financial management : journal of the Healthcare Financial Management Association. 2003;57(3):suppl 17 following 112.

16. Combes C, Meskens N, Rivat C, Vandamme JP. Using a KDD process to forecast the duration of surgery. International Journal of Production Economic. 2008;112(1):279-293.

17. Eijkemans MJ, van Houdenhoven M, Nguyen T, Boersma E, Steyerberg EW, Kazemier G. Predicting the unpredictable: a new prediction model for operating room times using individual characteristics and the surgeon's estimate. Anesthesiology. 2010;112(1):41-49.

18. Lee KL, Freiha F, Presti JC, Jr., Gill HS. Gender differences in radical cystectomy: complications and blood loss. Urology. 2004;63(6):1095-1099.

19. Yuh BE, Ciccone J, Chandrasekhar R, et al. Impact of previous abdominal surgery on robotassisted radical cystectomy. JSLS. 2009;13(3):398-405.

20. Kiran RP, Ahmed Ali U, Coffey JC, Vogel JD, Pokala N, Fazio VW. Impact of resident participation in surgical operations on postoperative outcomes: National Surgical Quality Improvement Program. Ann Surg. 2012;256(3):469-475.

21. Rogo-Gupta LJ, Lewin SN, Kim JH, et al. The effect of surgeon volume on outcomes and resource use for vaginal hysterectomy. Obstet Gynecol. 2010;116(6):1341-1347.

22. Pierorazio PM, Patel HD, Feng T, Yohannan J, Hyams ES, Allaf ME. Robotic-assisted versus traditional laparoscopic partial nephrectomy: comparison of outcomes and evaluation of learning curve. Urology. 2011;78(4):813-819.

23. Hayn $\mathrm{MH}$, Hussain $\mathrm{A}$, Mansour AM, et al. The learning curve of robot-assisted radical cystectomy: results from the International Robotic Cystectomy Consortium. Eur Urol. 2010;58(2):197-202.

24. Guru KA, Perlmutter AE, Butt ZM, et al. The learning curve for robot-assisted radical cystectomy. JSLS. 2009;13(4):509-514.

25. Stepaniak PS, Heij C, Mannaerts GH, de Quelerij M, de Vries G. Modeling procedure and surgical times for current procedural terminology-anesthesia-surgeon combinations and evaluation in terms of case-duration prediction and operating room efficiency: a multicenter study. Anesth Analg. 2009;109(4):1232-1245.

26. Silber JH, Rosenbaum PR, Even-Shoshan O, et al. Estimating anesthesia time using the medicare claim: a validation study. Anesthesiology. 2011;115(2):322-333. 
